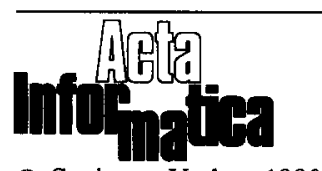

\title{
Referential Transparency, Definiteness and Unfoldability
}

\author{
Harald Søndergaard ${ }^{1}$ and Peter Sestoft ${ }^{2}$ \\ ${ }^{1}$ Department of Computer Science, University of Melbourne, Parkville 3052 Vic., Australia \\ 2 DIKU, University of Copenhagen, Universitetsparken 1, DK-2100 Copenhagen $\emptyset$, Denmark
}

Received November 30, 1987/January 4, 1990

\begin{abstract}
Summary. The term "referential transparency" is frequently used to indicate that a programming language has certain useful substitution properties. We observe, however, that the formal and informal definitions given in the literature are not equivalent and we investigate their relationships. To this end, we study the definitions in the context of a simple expression language and show that in the presence of non-determinism, the differences between the definitions are manifest. We propose a definition of "referential transparency", based on Quine's, as well as of the related notions: definiteness and unfoldability. We demonstrate that these notions are useful to characterize programming languages.
\end{abstract}

\section{Introduction}

The notions of referential transparency and referential opacity are common in discussions of properties of programming languages. They were originally suggested by Quine and brought into computer science by Landin and Strachey. The notions however have changed during time and the formal or informal definitions found in the literature are not equivalent.

The present paper discusses referential transparency, referential opacity, and similar notions. Precise definitions are suggested in the context of a non-deterministic expression language, but the perspective is broader since some of the definitions extend to programming languages more generally. The purpose is to clarify the relations between a number of properties of a formal language. These are very fundamental properties concerning identity and substitutivity, and so should be treated with great care.

The observation that in everyday language one may not always substitute a term by an equivalent term is exemplified by Quine. The statements

Cicero $=$ Tully

'Cicero' contains six letters

Offprint requests to: $\mathrm{H}$. Søndergaard 
are both true, but the replacement of the first name by the second will turn (1.2) false [13]. The point is that, owing to the quotes, 'Cicero' in (1.2) does not refer to the person Cicero, but to the word Cicero. In this way the quotes change or destroy reference, that is, the relation between a term and the object(s) it denotes.

The fact that words are sometimes used in this manner is noted by the medieval William of Sherwood, and Leibniz repeatedly mentions it (see Notes 1 and 2). Frege, in his discussion of sense and reference, gives the following formulation:

"It can also happen, however, that one wishes to talk about the words themselves or their sense. This happens, for instance, when the words of another are quoted. One's own words then first designate words of the other speaker, and only the latter have their usual reference. We then have signs of signs. In writing, the words are in this case enclosed in quotation marks. Accordingly, a word standing between quotation marks must not be taken to have its ordinary reference" (see Note 3 ).

A thorough investigation of the phenomenon has been undertaken by Quine. He calls a context referentially opaque if it destroys reference. Otherwise it is referentially transparent [14].

We just saw how quoting is referentially opaque, and the example

Tegucigalpa $=$ the capital of Honduras

Philip believes that Tegucigalpa is in Nicaragua

shows that "_ believes that _" is referentially opaque in place 2 , since (1.3) and (1.4) may well hold, whereas replacing Tegucigalpa in (1.4) by the right-hand side of (1.3) presumably yields a falsehood [13]. In general modal contexts fail to preserve reference, and so are referentially opaque.

The usefulness of Quine's notions to the science of programming languages was realized by Landin and Strachey. Strachey refers to Quine and the notion of referential transparency which

"means that if we wish to find the value of an expression which contains a sub-expression, the only thing we need to know about the sub-expression it its value" [18, page 16].

We shall henceforth refer to this principle as extensionality of the enclosing expression. Strachey also notes that

"We tend to assume automatically that the symbol $x$ in an expression such as $3 x^{2}+2 x+17$ stands for the same thing (or has the same value) on each occasion it occurs. This is the most important consequence of referential transparency" [18, page 22].

We shall refer to this principle as definiteness (of variables, which we thus call definite or indefinite depending on whether the principle applies or not).

Strachey's statement may be valid in the case of deterministic programming languages, but in a non-deterministic language it becomes a crucial question what is meant by "stands for the same thing". In a very natural interpretation it turns out that definiteness is not a consequence of extensionality. We demonstrate this by giving a language $Q_{1}$ which is referentially transparent in Quine's sense, but in which distinct occurrences of a variable (even within the same scope) may have different values. 
Based on Strachey's observations, Stoy gives an informal definition of referential transparency in this textbook on denotational semantics. Definiteness, which Strachey regards a consequence of referential transparency, becomes part of Stoy's definition:

"We use [referential transparency] to refer to the fact of mathematics which says: The only thing that matters about an expression is its value, and any subexpression can be replaced by any other equal in value. Moreover, the value of an expression is, within certain limits, the same whenever it occurs". [17, page 5]

This definition has three components. The first states the extensionality principle. The second is the principle that "any subexpression can be replaced by any other equal in value" which we refer to as Leibniz's law, or substitutivity of identity. The third expresses the principle of definiteness, and perhaps even some kind of determinacy. The qualification "within certain limits" is presumably to allow for the notion of scope of variables. Thus, in arguing that the lambda calculus is referentially transparent in his sense, Stoy notes that

"for $\ulcorner\lambda$ I.E $\urcorner$ all free occurrences of I in E denote the same value". [17, page 190, italics ours]

Stoy's book does not discuss mathematical semantics for non-deterministic languages, a topic that was just about to find its proper treatment at the time the book was written. So the above definition is perfectly adequate for Stoy's purpose. In general, however, it is advantageous to separate the component notions, because each provides a useful dimension in the characterization of a formal language, and because they are fundamentally distinct, as will be shown.

Languages with assignment lack many useful substitutivity properties. Consider

$$
x:=x-1 ; \quad y:=x .
$$

All occurrences of $x$ in (1.5) are in the same scope. A discussion of reference is complicated by the fact that an occurrence of $x$ on the left-hand side of $:=$ is interpreted rather differently to an occurrence of $x$ on the right-hand side. This is the well-known distinction between $\mathrm{L}$-values and $\mathrm{R}$-values of variables $[17,18]$. In (1.5), the first occurrence of $x$ is taken to denote a "location" which in turn "holds" a value, whereas the other occurrences denote values. Whether such ambiguity should count as destruction of reference may be a matter of taste. In any case variables are not definite, since even the two right-hand side occurrences of $x$ have different values.

In functional programming languages, the use of substitutivity of identity as a criterion for referential transparency has an added twist. Namely, one kind of substitution that may or may not apply is unfolding of a function application (corresponding to $\beta$-reduction in the lambda calculus). If unfolding is equivalence preserving, we say that unfoldability applies, or that $\beta$-reduction is admissible. The problem is that it is common to use the equality symbol " $=$ " when writing function definitions, as in

$$
f x=\ldots x \ldots
$$

This may be misleading, since it may well be the case that substitutivity of identity holds and yet unfoldability does not apply, so that it is not admissible to replace the function application $f e$ by the instantiated right-hand side ...e... of (1.6). This 
will be exemplified when we investigate unfoldability and its relation to the notions of referential transparency and definiteness.

Textbooks on programming languages offer a variety of suggestions as to what referential transparency means. The following is a selection: definiteness (Note 4), absence of side-effects (Note 5), determinacy (Note 6), unfoldability (Note 7), extensionality (Note 8), and applicability of Leibniz's law (Note 9). This variety is justified to some extent because textbooks often teach more efficiently by simplifying issues slightly. It must however confuse a student seeking precise knowledge: certainly not all of the above notions are equivalent. The intention with this paper is to clarify some of their relations.

After some preliminary remarks on notation in Sect. 2, we present in Sect. 3 a simple expression language called $Q_{0}$. This language will serve as a basis for the discussion of concepts. In Sect. 4 we define the notions of referential transparency, referential opacity, definiteness, and unfoldability, and we use these to characterize the language $Q_{0}$. In Sect. 5 we apply some distortions to the semantics of the language, thus obtaining a series of slightly different languages $Q_{1}-Q_{4}$ that exhibit varying combinations of the properties introduced in Sect. 4. We summarize in Sect. 6 and draw conclusions as to the relations between the notions discussed in the paper.

\section{Preliminaries}

The present note is concerned with issues of reference. We should therefore state exactly which devices the paper itself employs to indicate referential use. We use italics to indicate identifiers such as $x$ or exp. We use the pair of brackets $\llbracket \ldots \rrbracket$ for quasi-quotation of expressions: meta-language variables that appear between the brackets denote whatever they are bound to, whereas all other symbols denote themselves. This is the usual convention of denotational semantics. Thus the brackets are nothing but "Quine corners" [12].

To distinguish various kinds of equivalence, we use two equivalence signs: a "syntactic" and a "semantic" one. The symbol $\equiv$ is used between expressions to denote syntactic identity, that is, the relation in which an expression stands to itself and to no other expression. Trivial as it may seem, there is good use for $\equiv$, because we use variables and expressions that range over syntactic objects (expressions); thus it makes sense to write, for instance, $e_{1} \equiv e_{2}$, where $e_{1}$ and $e_{2}$ are meta-language variables.

The equality symbol, $=$, is used for a number of purposes: (1) between elements of a set, (2) between sets, with the usual meaning: each is a subset of the other, (3) between functions to denote strong pointwise equality, and (4) between expressions to denote codenotation: both sides denote the same object.

In the following, some knowledge of denotational semantics will be beneficial [17]. A note concerning the modeling of non-determinism and the interpretation of equality is in order. Consider the non-deterministic expressions $e_{1}$ and $e_{2}$. We follow the standard approach and let the denotation of an expression be the set of values it may evaluate to (including possible error values). So $e_{1}=e_{2}$ means that whatever $e_{1}$ may evaluate to, $e_{2}$ may evaluate to, and vice versa. This, however, does not mean that $e_{1}$ and $e_{2}$ will necessarily evaluate to the same thing. In this way the introduction of non-determinism highlights a difference between denotation and intended meaning: expression $e$ may denote a set, but the intended meaning is that $e$ stands for some member of the set, we just cannot know which. 


\section{A Simple Expression Language}

This section introduces the language $Q_{0}$ which we shall use for defining and illustrating various substitution properties. $Q_{0}$ is a very restricted expression language and certainly useless as a programming language. Even so, we feel justified in basing our analysis on it, because it incorporates constructs known from existing programming languages, albeit only those considered relevant for our discussion: Occam's razor has been applied whenever possible, to bring the issues under investigation into focus. The syntax of $Q_{0}$ is given in Table 1 . In Sect. 5 we discuss a series of languages $Q_{1}-Q_{4}$, all having the same syntax as $Q_{0}$ but with differing semantics. Each of these semantics is defined by changing the definition of the preceding language very slightly. Nevertheless they will be shown to lead to rather different substitution properties.

The "full form" displayed will help certain definitions, but for readability we normally use the "short form" of expressions, using parentheses to remove ambiguity. However, we use $\lambda_{x} e e^{\prime}$ simply, for $\left(\lambda_{x} e\right) e^{\prime}$, in the understanding that variables in $e^{\prime}$ are not bound by the occurrence of $\lambda_{x}$. Since $x$ is the only available variable, the standard notation " $\lambda x . e$ " for a lambda abstraction would be pleonastic, and the use of " $\lambda e$ " would disturb intuition, hence the notation " $\lambda_{x} e$ ".

Table 2 gives a denotational definition of $Q_{0}$. The set of values of expressions is

$$
V=\{0,1, \#\} \text {, }
$$

Table 1. Syntax for the $Q$ family of languages

\begin{tabular}{|c|c|c|}
\hline & Full form & Short form \\
\hline $\exp \rightarrow$ & & 0 \\
\hline 1 & 1 & 1 \\
\hline i & $x$ & $x$ \\
\hline I & $\langle$ minus exp exp $\rangle$ & $\exp -\exp$ \\
\hline i & $\langle$ numeral? exp $\rangle$ & @exp \\
\hline i & $\langle$ choose exp exp & $\exp \sqcap \exp$ \\
\hline i & $\langle$ apply fun exp $\rangle$ & fun $\exp$ \\
\hline fun $\rightarrow$ & $\langle l a m b d a \exp \rangle$ & $\lambda_{x} \exp$ \\
\hline
\end{tabular}

Table 2. Semantics of $Q_{0}$

Below, $e, e_{1}, e_{2} \in \exp ; f \in f u n ; v_{1}, v_{2} \in V ; u \in P V$.

$$
\begin{array}{ll}
D \llbracket e \rrbracket & =E \llbracket e \rrbracket\{\#\} \\
E \llbracket 0 \rrbracket u & =\{0\} \\
E \llbracket 1 \rrbracket u & =
\end{array}
$$


where \# signifies failure of evaluation. The non-determinism of expressions is modeled by having the semantic equations define sets of possible results. We therefore use a semantic domain

$$
P V=(\mathscr{P} V) \backslash\{\varnothing\},
$$

that is, the set of non-empty subsets of $V$. The empty set is excluded because an expression must have a value (if evaluation fails, then its "value" is \#). The valuation function for expressions is

$$
D: \exp \rightarrow P V
$$

which in turn is defined in terms of the functions

$$
\begin{aligned}
& E: \exp \rightarrow P V \rightarrow P V \\
& F: f u n \rightarrow P V \rightarrow P V .
\end{aligned}
$$

An expression is evaluated given an element from $P V$ (namely the set of possible values of $x$ ), to yield an element from $P V$ (namely the set of possible result values).

Let us informally describe evaluation of compound expressions. If $e_{1}$ and $e_{2}$ may evaluate to $v_{1}$ and $v_{2}$, respectively, then $e_{1}-e_{2}$ may evaluate to $v_{1}-v_{2}$ (which fails in case $v_{1}<v_{2}$ ). If $e$ is a numeral ( 0 or 1$)$, then @ $e$ must evaluate to 1 , otherwise it must evaluate to 0 . The choice expression $e_{1} \sqcap e_{2}$ may evaluate to $v$ if either $e_{1}$ may or $e_{2}$ may, and it may fail if either $e_{1}$ may or $e_{2}$ may. This amounts to erratic non-determinism [4]. Finally, let $u$ be the set of values to which $e^{\prime}$ may evaluate. The expression $\lambda_{x} e e^{\prime}$ may evaluate to whatever $e$ may evaluate to, given that an occurrence of $x$ in $e$ must evaluate to some $v \in u$. There are, however, two provisos, given by (F1): (1) if evaluation of $e^{\prime}$ must fail, then so must evaluation of $\lambda_{x} e e^{\prime}$, and (2) if evaluation of $e^{\prime}$ may fail, then so may evaluation of $\lambda_{x} e e^{\prime}$. This yields the natural counterpart to applicative order evaluation in a deterministic language.

We can now define semantic equivalence of expressions as codenotation. Expressions are semantically equivalent iff they have the same denotation. By definition,

$$
e_{1}=e_{2} \quad \text { iff } D \llbracket e_{1} \rrbracket=D \llbracket e_{2} \rrbracket
$$

for all $e_{1}, e_{2} \in \exp$. Note that $D$ is a parameter in this definition, in the sense that the definition applies throughout the paper, even when $D$ (or $E$ and $F$, on which $D$ depends) is changed.

It is a crucial feature of $Q_{0}$ that $x$ becomes bound to a (non-empty) set of values. As a consequence, the denotation of, say, $\lambda_{x}(x-x)(0 \sqcap 1)$ is $\{0,1$, \# $\}$ rather than $\{0\}$. In other words, even within the same scope, distinct occurrences of a variable need not evaluate to the same result, witness the subexpression $(x-x)$ in $\lambda_{x}(x-x)$ $(0 \sqcap 1)$. This type of semantics is known as plural [5] or run time choice [8] semantics. The last term hints at the operational view that a value for $x$ is chosen anew every time $x$ is met during evaluation.

The language $Q_{0}$ lacks a number of substitutivity properties which are introduced in the next section. In Sect. 5 we investigate the consequences of various changes in the semantic definition with respect to such properties, and in Sect. 6 we summarize and compare the properties. Elsewhere we have discussed further (algebraic) properties of languages akin to $Q_{0}-Q_{4}$, but with recursion $[15,16]$. 


\section{Referential Transparency and Substitution Properties}

Quine defines referential transparency using the concept of a purely referential position in a sentence: "the position must be subject to the substitutivity of identity" [14, page 142]. He then defines referential transparency:

"I call a mode of containment $\phi$ referentially transparent if, whenever an occurrence of a singular term $t$ is purely referential in a term or sentence $\psi(t)$, it is purely referential also in the containing term or sentence $\phi(\psi(t))$ " [14, page 144].

So $\phi$ is referentially transparent if it preserves pure referentiality, that is, preserves substitutivity of identity. This amounts to Leibniz's law, and to extensionality. However, it is independent of the other important substitutivity properties we have met. We now define the various substitutivity concepts more precisely and check whether they apply to $Q_{0}$.

A position $p$ is a (possibly empty) sequence of natural numbers, $p \in N^{*}$. The empty sequence is denoted by $\varepsilon$. The sequence constructor is denoted by ".". Let $\Omega$ be an operator. Expression $e$ with $e^{\prime}$ inserted at position $p, e\left[e^{\prime} / p\right]$, is defined by

$$
\begin{aligned}
& e\left[e^{\prime} / \varepsilon\right] \equiv e^{\prime} \\
& e\left[e^{\prime} / i \cdot p\right] \equiv\left\langle\Omega e_{1} \ldots e_{i}\left[e^{\prime} / p\right] \ldots e_{n}\right\rangle \quad \text { if } e \equiv\left\langle\Omega e_{1} \ldots e_{i} \ldots e_{n}\right\rangle, \quad \text { else undefined. }
\end{aligned}
$$

Position $p$ is purely referential in expression $e$ iff $e_{1}=e_{2} \Rightarrow e\left[e_{1} / p\right]=e\left[e_{2} / p\right]$ for all $e_{1}, e_{2} \in \exp$. That is, a position is purely referential in expression $e$ iff it is subject to the substitutivity of identity. Note that for every expression $e$, position $\varepsilon$ is purely referential in $e$. An operator $\Omega$ is referentially transparent in place $i$ iff for every expression $e \equiv\left\langle\Omega e_{1} \ldots e_{i} \ldots e_{n}\right\rangle$, whenever position $p$ is purely referential in $e_{i}$, position $i \cdot p$ is purely referential in $e$. Otherwise $\Omega$ is referentially opaque in place $i$. We shall also say that $\Omega$ is referentially transparent, simply, iff it is referentially transparent in all places. Similarly, $\Omega$ is referentially opaque iff it is referentially opaque in some place. Finally, we call a formal language referentially transparent if all its operators are referentially transparent; otherwise we call it referentially opaque.

Examples of referentially opaque operators are@ as introduced in Sect. 3, Pascal's quotation marks used for character strings, and Lisp's quote (a few other operators from the Lisp category of "fexpr" are referentially opaque, though not all: cond, for example, is referentially transparent). Common to these operators is that they depend on the form of their argument rather than its value. Note that a position may well be purely referential globally and yet not locally: if (as in Lisp) we had had an eval and a quote operator such that eval (quote $e$ ) $=e$, then the position of $e$ in eval (quote $e$ ) would be purely referential, in spite of the fact that quote is referentially opaque.

A language has the definiteness property iff the denotation of a variable necessarily is a single value. For a language in our family, the implication of this is that $x-x$ will always evaluate to 0 (or possibly \#), even though $x$ may be bound (by a function application) to an expression such as $0 \sqcap 1$. To see that this is to give $x$ a special treatment, consider replacing $x$ by what it is bound to. This yields $(0 \sqcap 1)-(0 \sqcap 1)$, which may evaluate to either 0 or 1 , or fail. If definiteness applies, we say that the variables are definite, otherwise we call them indefinite.

Let $e\left[e^{\prime}\right]$ denote the expression $e$ with all free occurrences of $x$ replaced by $e^{\prime}$ (even when enclosed by semantic brackets as in $\llbracket e\left[e^{\prime}\right] \rrbracket$ ). We say that unfoldability applies (for a language in our family) iff $\lambda_{x} e e^{\prime}=e\left[e^{\prime}\right]$ for all $e, e^{\prime} \in \exp$. 
Let us analyse $Q_{0}$ in terms of the introduced notions. First, $Q_{0}$ is not referentially transparent, because the operator @ destroys reference. As an example, $1-0=1$ since both sides denote $\{1\}$. However, @ $(1-0) \neq @ 1$ since the left-hand side denotes $\{0\}$, whereas the right-hand side denotes $\{1\}$. All other operators are referentially transparent, as will be shown in Sect. 5.1. Second, variables in $Q_{0}$ are indefinite. Thus the $Q_{0}$ expression

$$
\lambda_{x}(x-x)(0 \sqcap 1)
$$

denotes $\{0,1, \#\}$ rather than $\{0\}$. Finally, unfoldability does not apply in the case of $Q_{0}$. To see this, consider the expression

$$
\lambda_{x} 0(0-1)
$$

Unfolding this application yields 0 , but $D \llbracket \lambda_{x} 0(0-1) \rrbracket=\{\#\}$, while $D \llbracket 0 \rrbracket=\{0\}$.

\section{Variations on a Theme}

In this section various changes are made to the semantics of $Q_{0}$. We thereby obtain a series of languages $Q_{1}-Q_{4}$ that exhibit quite different substitutivity properties. In this way the languages illustrate how the properties interrelate.

\subsection{Obtaining Referential Transparency}

We have seen that @ is referentially opaque. The definition of the semantic function $E$ is now changed slightly to make @ void of effect. We replace (E1) by

$$
E \llbracket @ e \rrbracket u=E \llbracket e \rrbracket u .
$$

The resulting language is called $Q_{1}$.

Example. The $Q_{1}$ expression@(@) denotes $\{1\}$, whereas qua $Q_{0}$ expression it denotes $\{0\}$.

Clearly, by (E1'), @ is no longer referentially opaque. In fact the following proposition holds.

Proposition. $Q_{1}$ is referentially transparent.

Proof. Consider the semantic definition in Table 2. The result of applying $E$ to expressions of the form $\left(\lambda_{x} e_{1}\right) e_{2}, e_{1} \sqcap e_{2}$, or $e_{1}-e_{2}$ only depends on the meaning $E \llbracket e_{i} \rrbracket u$ of the subexpressions $e_{i}$ for $i \in\{1,2\}$. We demonstrate this in the case of $e_{1}$ in expression $e_{1} \sqcap e_{2}$. If $e_{1}=e_{1}^{\prime}$, then $E \llbracket e_{1} \rrbracket u=E \llbracket e_{1}^{\prime} \rrbracket u$, by definition of " $="$ on expressions. So for $u \in P V, E \llbracket e_{1} \sqcap e_{2} \rrbracket u=E \llbracket e_{1} \rrbracket u \cup E \llbracket e_{2} \rrbracket u=E \llbracket e_{1}^{\prime} \rrbracket u \cup E \llbracket e_{2} \rrbracket u$ $=E \llbracket e_{1}^{\prime} \sqcap e_{2} \rrbracket u$, and so $e_{1} \sqcap e_{2}=e_{1}^{\prime} \sqcap e_{2}$. This proves that $(-\sqcap-)$ is purely referential in place 1 , and since $e_{1}$ and $e_{2}$ were arbitrary, it follows that it is referentially transparent in place 1 . Similar arguments hold for all other cases.

Although no operator thus destroys reference, variables are still not definite. Namely, example (4.1) qua $Q_{1}$ expression evaluates exactly like before, since it does not contain 
the @ operator. Furthermore, as example (4.2) qua $Q_{1}$ expression shows, unfoldability still does not apply.

This proves that a non-deterministic programming language may well be referentially transparent in the sense of Sect. 4 (and of Quine) and yet violate the principle that "a variable stands for the same thing on each occasion it occurs". It also shows that referential transparency does not imply unfoldability.

\subsection{Obtaining Unfoldability}

We now modify $Q_{1}$ to make unfolding equivalence preserving. This is done by replacing (F1) in the definition of $Q_{1}$ by

$$
F \llbracket \lambda_{x} e \rrbracket u=E \llbracket e \rrbracket u .
$$

The resulting language is called $Q_{2}$. Evaluation in $Q_{2}$ resembles normal order evaluation in deterministic languages.

Example. The $Q_{2}$ expression $\lambda_{x} 0(0-1)$ denotes $\{0\}$, whereas qua $Q_{0}$ or $Q_{1}$ expression it denotes $\{\#\}$, that is, evaluation must fail.

Like $Q_{1}, Q_{2}$ is referentially transparent, and variables in $Q_{2}$ are still indefinite, as witnessed by (4.1) qua $Q_{2}$ expression. However, $Q_{2}$ allows for unfolding as the following proposition shows.

Proposition. Let $e$ and $e^{\prime}$ be $Q_{2}$ expressions. Then $\lambda_{x} e e^{\prime}=e\left[e^{\prime}\right]$.

Proof. Let $u \in P V$ and let $u^{\prime}=E \llbracket e^{\prime} \rrbracket u$. Since by (E 3$)$ and (F $\left.1^{\prime}\right)$,

$$
E \llbracket \lambda_{x} e e^{\prime} \rrbracket u=F \llbracket \lambda_{x} e \rrbracket\left(E \llbracket e^{\prime} \rrbracket u\right)=E \llbracket e \rrbracket u^{\prime},
$$

it suffices to show that $E \llbracket e \rrbracket u^{\prime}=E \llbracket e\left[e^{\prime}\right] \rrbracket u$ for all $e, e^{\prime} \in \exp$. We show this by induction on the structure of $e$. The cases $e \equiv 0$ and $e \equiv 1$ are trivial. For $e \equiv x$ we have: $E \llbracket x \rrbracket u^{\prime}=E \llbracket e^{\prime} \rrbracket u=E \llbracket x\left[e^{\prime}\right] \rrbracket u$. The case $e \equiv e_{1}-e_{2}$ is similar to $e_{1} \sqcap e_{2}$ below.

Case $e \equiv @ e_{1}$ :

$$
\begin{aligned}
E \llbracket @ e_{1} \rrbracket u^{\prime} & =E \llbracket e_{1} \rrbracket u^{\prime} & & \text { by }\left(\mathrm{E} 1^{\prime}\right) \\
& =E \llbracket e_{1}\left[e^{\prime}\right] \rrbracket u & & \text { by the induction hypothesis } \\
& =E \llbracket @\left(e_{1}\left[e^{\prime}\right]\right) \rrbracket u & & \text { by }\left(\mathrm{E} 1^{\prime}\right) \\
& =E \llbracket\left(\left(e_{1}\right)\left[e^{\prime}\right] \rrbracket u\right. & & \text { q.e.d. }
\end{aligned}
$$

Case $e \equiv e_{1} \sqcap e_{2}$ :

$$
\begin{aligned}
E \llbracket e_{1} \sqcap e_{2} \rrbracket u^{\prime} & =E \llbracket e_{1} \rrbracket u^{\prime} \cup E \llbracket e_{2} \rrbracket u^{\prime} & & \text { by }(E 2) \\
& =E \llbracket e_{1}\left[e^{\prime}\right] \rrbracket u \cup E \llbracket e_{2}\left[e^{\prime}\right] \rrbracket u & & \text { by the induction hypothesis } \\
& =E \llbracket e_{1}\left[e^{\prime}\right] \sqcap e_{2}\left[e^{\prime}\right] \rrbracket u & & \text { by (E2) } \\
& =E \llbracket\left(e_{1} \sqcap e_{2}\right)\left[e^{\prime}\right] \rrbracket u & & \text { q.e.d. }
\end{aligned}
$$

Case $e \equiv \lambda_{x} e_{1} e_{2}$ :

$$
\begin{aligned}
E \llbracket \lambda_{x} e_{1} e_{2} \rrbracket u^{\prime} & =F \llbracket \lambda_{x} e_{1} \rrbracket\left(E \llbracket e_{2} \rrbracket u^{\prime}\right) & & \text { by }(\mathrm{E} 3) \\
& =F \llbracket \lambda_{x} e_{1} \rrbracket\left(E \llbracket e_{2}\left[e^{\prime}\right] \rrbracket u\right) & & \text { by the induction hypothesis } \\
& =E \llbracket \lambda_{x} e_{1}\left(e_{2}\left[e^{\prime}\right]\right) \rrbracket u & & \text { by }(\mathrm{E} 3) \\
& =E \llbracket\left(\lambda_{x} e_{1} e_{2}\right)\left[e^{\prime}\right] \rrbracket u & & \text { q.e.d. }
\end{aligned}
$$

By structural induction, $E \llbracket e \rrbracket u^{\prime}=E \llbracket e\left[e^{\prime}\right] \rrbracket u$, and the proposition follows. 


\subsection{Obtaining Definiteness at the Expense of Unfoldability}

All of the languages so far had indefinite variables: different occurrences of a variable might evaluate differently, so for instance $x-x$ might evaluate to 1 . We now want to make variables definite. This can be done by making the semantics singular [5], that is, by guaranteeing that at any instant, a variable denotes exactly one value (rather, a singleton set of values). This type of semantics is found also under the label call time choice semantics, reflecting the operational view that the value for a (lambda) variable is determined once: at the time of application [8]. More precisely we replace line (E3) in the definition of $Q_{2}$ by

$$
E \llbracket f e \rrbracket u=\cup\{F \llbracket f \rrbracket\{v\} \mid v \in E \llbracket e \rrbracket u\} .
$$

Here $U$ is the distributed union operator. The resulting language is called $Q_{3}$.

Example. The $Q_{3}$ expression

$$
\lambda_{x}(x-x)(0 \sqcap 1)
$$

denotes $\{0\}$, whereas qua $Q_{0}, Q_{1}$, or $Q_{2}$ expression it denotes $\{0,1$, \#\}.

It should be clear that $x$ is now definite. However, as compared to $Q_{2}, Q_{3}$ has lost unfoldability. Unfolding the call in (5.1) yields $(0 \sqcap 1)-(0 \sqcap 1)$ which denotes $\{0,1, \#\}$.

In the presence of non-determinism, we cannot obtain both definiteness and unfoldability. If we retain $(\mathrm{F} 1)$ rather than $\left(\mathrm{F} 1^{\prime}\right)$, the resulting language (let us call it $Q_{3}^{\prime}$ ) will still have definite variables and lack unfoldability, since the above example still applies. Usually designers of non-deterministic languages choose to renounce unfoldability in order to keep variables definite. This is the case, for example, in the wide-spectrum language CIP-L whose transformation system explicitly requires a function to be determinate for any of its applications to be unfolded [3, page 99].

\subsection{Giving Up Non-Determinism}

The only way to achieve both definiteness and unfoldability is to give up nondeterminism. This can be done for instance by replacing (E2) in the definition of $Q_{3}$ by

$$
E \llbracket e_{1} \sqcap e_{2} \rrbracket u=E \llbracket e_{1} \rrbracket u .
$$

The resulting language is called $Q_{4}$.

Example. The $Q_{4}$ expression $0 \sqcap 1$ denotes $\{0\}$, whereas qua $Q_{0}, Q_{1}, Q_{2}$, or $Q_{3}$ expression it denotes $\{0,1\}$.

Thus $\sqcap$ is now almost void of effect. All sets that are returned by $E$ are singleton sets, and it should be clear that both definiteness and unfoldability apply. Note that $Q_{4}$ is still referentially transparent, as is $Q_{1}, Q_{2}$, and $Q_{3}$. The operator $\sqcap$ does not destroy reference by ignoring $e_{2}$. It is still true that "all that matters about $e_{2}$ is its value", although this value is not being used for anything. 


\section{Conclusion}

We have suggested a definition of referential transparency in programming languages which is in accordance with Quine's notion [14]. By this, an operator is referentially transparent if it preserves applicability of Leibniz's law, or substitutivity of identity: the principle that any subexpression can be replaced by any other equal in value.

Even though definiteness (the principle that within the same scope, distinct occurrences of a variable evaluate to the same) is sometimes taken as the definition of referential transparency, a language may well lack definiteness and yet be referentially transparent. The same goes for unfoldability, or admissibility of $\beta$-reduction. Neither of these properties are implied by referential transparency. This has been exemplified by a series of languages $Q_{0}-Q_{4}$. It was also argued that in the presence of nondeterminism it is impossible to obtain both definiteness and unfoldability at the same time. However, a non-deterministic language may well be referentially transparent.

Table 3 summarizes these findings. The table does not present an exhaustive list of the possible combinations of "yes" and "no". Many more (and indeed more contrived) languages are possible. So one should not deduce from the table that definiteness implies referential transparency or that determinacy implies unfoldability, for example. Such implications fail to hold in general. We conclude that it is useful to separate the issues: determinacy, definiteness, unfoldability, and referential transparency, because they cover different aspects of languages, and they all provide useful dimensions along which to characterize programming languages.

We have avoided such classical terms for evaluation strategies as "call by value" and "call by name", simply because they were not defined for non-deterministic languages. Table 4 suggests a natural way to generalize the notions (we find the nomenclature more natural than Clinger's [5], as well as in better agreement with current use for deterministic languages). In the absence of non-determinism, the two rows of Table 4 collapse into one: at least in a language without side-effects, there is no difference in the results obtained using call by name and call by need.

Table 3. Properties of the languages

\begin{tabular}{lccccc}
\hline & $Q_{0}$ & $Q_{1}$ & $Q_{2}$ & $Q_{3}$ & $Q_{4}$ \\
\hline Determinacy & no & no & no & no & yes \\
Definiteness & no & no & no & yes & yes \\
Unfoldability & no & no & yes & no & yes \\
Referential transparency & no & yes & yes & yes & yes \\
\hline
\end{tabular}

Table 4. Relations to operational notions

\begin{tabular}{lll}
\hline & "Applicative" order & "Normal" order \\
\hline $\begin{array}{lll}\text { Plural semantics } \\
\text { (run time choice) }\end{array}$ & $Q_{0}$ and $Q_{1}$ & $Q_{2}$ \\
$\begin{array}{l}\text { Singular semantics } \\
\text { (call time choice) }\end{array}$ & $\left(Q_{3}^{\prime}\right)$ & Call by name \\
\hline
\end{tabular}


We have not discussed side-effects in the present paper, since this phenomenon does not come up in purely applicative languages. In a language like Algol 60, whose semantics can only be explained in terms of a notion of "state" [17], the expression $x-x$ (where $x$ is an integer variable) may well evaluate to 6 , say, owing to side-effects of the evaluation of $x$. Clearly non-determinism is not the only obstacle for definiteness.

Acknowledgements. We have found the referees' critical comments very helpful when revising this paper. In particular, the present simplified and improved example language is basically due to one of the referees. Thanks also go to Rodney Topor and Phil Wadler. The first author has been supported in part by the Danish Research Academy, the second by the Danish Natural Science Research Council.

\section{Notes}

1. "A $\infty$ B significat A et B esse idem, seu ubique sibi posse substitui. (Nisi prohibeatur, quod fit in iis, ubi terminus aliquis certo respectu considerari declaratur ver. $\mathrm{g}$. licet trilaterum et triangulum sint idem, tamen si dicas triangulum, quatenus tale, habet 180 gradus; non potest substitui trilaterum. Est in eo aliquid materiale)." [6, page 261$]$.

2. "Au reste il arrive quelques fois que nos idées et pensées sont la matiere de nos discours et font la chose même qu'on veut signifier, et les notions reflexives entrent plus qu'on ne croit dans celles des choses. On parle mêmes quelque fois des mots materiellement sans que dans cet endroit là precisement, on puisse substituer à la place du mot la signification, ou le rapport aux idées ou aux choses" [11, page 287].

3. „Es kann aber auch vorkommen, daß man von den Worten selbst oder von ihrem Sinne reden will. Jenes geschieht, z.B., wenn man die Worte eines Andern in gerader Rede anführt. Die eigenen Worte bedeuten dann zunächst die Worte des Andern und erst diese haben die gewöhnliche Bedeutung. Wir haben dann Zeichen von Zeichen. In der Schrift schließt man in diesem Falle die Wortbilder in Anführungszeichen ein. Es darf also ein in Anführungszeichen stehendes Wortbild nicht in der gewöhnlichen Bedeutung genommen werden “ [7, page 28].

4. "Program variables necessarily violate Quine's (1960) request for "referential transparency": that a "variable" (=a designation) has the same value at every position in the text" [2, page 353].

5. "The purpose of an expression is to compute a value. Expressions are composed of operands such as constants and variables, operators and possibly function calls. The process of evaluation is to substitute values for the operands and perform the operations. The values which are associated with the variables of a program form the state space or environment of the program. Evaluation of an expression should only produce a value and not change the environment. This idea is called by the grandiose name referential transparency" [10, page 93].

6. "One essential ingredient of functional programming is that the value of a function is determined solely by the values of its arguments. Thus, calls of the function using the same arguments will always produce the same value. This property is called referential transparency" [10, page 357].

7. “... consider a logic program containing two statements

$\mathrm{S} 1$ : parent $(x, z)$ if mother $(x, z)$

$S 2$ : grandparent $(x, y)$ if parent $(x, z)$, parent $(z, y)$ 
We may ... substitute for parent $(x, z)$ in $S 2$ its referent $\operatorname{mother}(x, z) \ldots$ to obtain

\section{S3: grandparent $(x, y)$ if mother $(x, z)$, parent $(z, y)$}

... Logic and other pure declarative languages therefore enjoy what is called referential transparency; what any of their constituents refer to in a program can be ascertained by exploiting the soundness of substitutivity, taking no account of the irrelevant run-time context" [9, page 240-241].

8. "... a principle known as referential transparency [Quine 1960] that holds in mathematics: The value of an expression can be determined solely from the values of its subexpressions, and if any subexpression is replaced by an arbitrary expression with the same value then the value of the entire expression remains unchanged" [19, page 29].

9. "A language that supports the concept that "equals can be substituted for equals" without changing the values of expressions is said to be referentially transparent" [1, page 178].

\section{References}

1. Abelson, H., Sussman, G.J.: Structure and interpretation of computer programs. Cambridge, Mass.: MIT Press 1985

2. Bauer, F.L., Wössner, H.: Algorithmic language and program development. Berlin Heidelberg New York: Springer 1982

3. Bauer, F.L. et al. The Munich project CIP, vol. I: The wide spectrum language CIP-L (Lect. Notes Comput. Sci., vol. 183). Berlin Heidelberg New York: Springer 1985

4. Broy, M.: A theory for nondeterminism, parallelism, communication, and concurrency. Theor. Comput. Sci. 45, 1-61 (1986)

5. Clinger, W.: Nondeterministic call by need is neither lazy nor by name. Proc. 1982 Symp. Lisp Funct. Progr., Pittsburgh, Pennsylvania pp. 226-234, 1982

6. Couturat, L.: Opuscules et fragments inédits de Leibniz. Hildesheim: Georg Olm 1961

7. Frege, G.: Über Sinn und Bedeutung. Z. Philosophie philosophische Kritik 100, 25-50 (1892). English translation in: Geach, P., Black, M. (eds.) Translations from the philosophical writings of Gottlob Frege, pp. 56-78. Oxford: Blackwell 1952

8. Hennessy, M.C.B., Ashcroft, E.A.: Parameter-passing mechanisms and nondeterminism. Proc. Ninth ACM Symp. Theory Comput., pp. 306-311. Boulder, Colorado 1977

9. Hogger, C.J.: Introduction to logic programming. London: Academic Press 1984

10. Horowitz, E.: Fundamentals of programming languages. Berlin Heidelberg New York: Springer 1983

11. Leibniz, G.W.: Nouveaux essais sur l'entendement humain, III, 2. In: Gottfried Wilhelm Leibniz - Sämtliche Schriften und Briefe VI, 6. Berlin (GDR): Akademie-Verlag 1962

12. Quine, W.V.O.: Mathematical logic. New York: Norton 1940

13. Quine, W.V.O.: Reference and modality. In: From a logical point of view, pp. 139-159. Cambridge, Mass.: Harvard University Press 1953

14. Quine, W.V.O.: Word and object. Cambridge, Mass: MIT Press 1960

15. Søndergaard, H., Sestoft, P.: Non-determinism in functional languages. Comput. J. (to appear)

16. Søndergaard, H., Sestoft, P.: Referential transparency and allied notions. University of Copenhagen, Denmark, DIKU Research Report 88/7, 1988

17. Stoy, J.E.: Denotational semantics. Cambridge, Mass.: MIT Press 1977

18. Strachey, C.: Fundamental concepts in programming languages. Int. Summer School in Computer Programming, Copenhagen, Denmark 1967 (unpublished)

19. Waite, W.M., Goos, G.: Compiler construction. Berlin Heidelberg New York: Springer 1984 